\title{
Analysis of major Fusarium toxins and their retention during processing
}

\author{
Masayo KUSHIRO * \\ Headquarters, NARO, 3-1-1 Kannondai, Tsukuba 305-8517, Japan
}

Key words : deoxynivalenol; fumonisin; Fusarium; milling; nivalenol, rice

(Received: June 9, 2013)

\begin{abstract}
Fusarium species are plant pathogenic fungi commonly found in the field. Wet and temperate weather during the growth of grain plants often results in diseases caused by several Fusarium species, which cause two forms of agricultural damage: a reduction in harvest (shriveled grains) and a threat to food safety (contamination of grains by mycotoxins). Since 2001, I have been involved in the development of analytical methods for major Fusarium toxins that can potentially pollute crop grains (especially rice and wheat) and the investigation of the retention of these toxins during processing, such as milling. In this review, I will present two topics; "Detection of fumonisins in rice" and "Retention of deoxynivalenol and nivalenol during milling of wheat".
\end{abstract}

\section{Topic I : Detection of fumonisins in rice}

\section{Introduction}

Rice is one of the major staple crops worldwide, feeding about half the world population ${ }^{1)}$. Fungal infection and mycotoxin contamination in rice is less reported than that in wheat and corn; however, some Fusarium fungi occasionally infect rice in the paddy field ${ }^{2)}$. Rice is susceptible to a Fusarium fungus, Fusarium/Gibberella fujikuroi, which causes disorders called "Bakanae" disease in rice plants with a symptom of unusually large height. Taxonomically, F. fujikuroi is a member of the G. fujikuroi species complex and is in the mating population (MP)- $\mathrm{C}^{3)}$.

Fumonisins are Fusarium toxins and are among the most important mycotoxins with regards to food and feed safety ${ }^{4}$. They are produced mainly by Fusarium verticillioides and F. proliferatum, which often damage corn kernels worldwide ${ }^{5)}$. Both $F$. verticillioides and F. proliferatum are members of the G. fujikuroi species complex, as MP-A and MP-D, respectively ${ }^{3)}$. The most abundant analogue in nature is fumonisin B1 (FB1), followed by fumonisin B2 (FB2) and then fumonisin B3 (FB3) ${ }^{6}$. FB1 has been implicated in causing leukoencephalomalacia in horses, pulmonary oedema syndrome in pigs, and hepatotoxicity and hepatocellular carcinogenicity in rats $^{7}$. Concerning human toxicity, the International Agency for Research on Cancer (IARC)

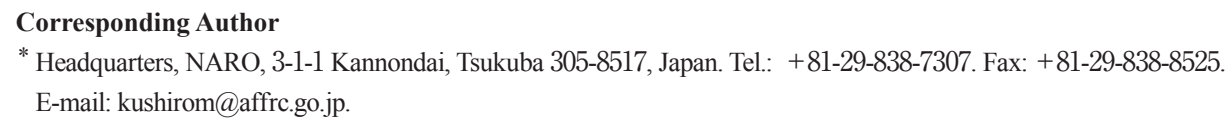


evaluated FB 1 derived from $F$. verticillioides as Group 2 B, i.e. a possible human carcinogen ${ }^{8)}$. In addition, fumonisins have been suggested as a potential cause of neural tube defects in human babies since first reported in 1999 among a high corn-consuming population in the United States (Texas)-Mexico border area ${ }^{9}$. In the European Union, regulatory limits of $0.2 \mathrm{ppm}$ to $4 \mathrm{ppm}$ have been set, depending on the type of food.

So far, corn and corn-based products are the only commodities that are known to potentially contain significant amounts of fumonisins. However, considering the fact that rice adherent fungus G. fujikuroi is taxonomically close to $F$. verticillioides and $F$. proliferatum, there exists a potential risk of fumonisin contamination in rice. Furthermore, there are sporadic reports of low-level contamination of fumonisins in rice in the United States ${ }^{10}$. Although the contents of fumonisins in rice were around or below $1 \mathrm{ppm}$, the monitoring of fumonisins in rice should be required to assess the exposure to fumonisins in populations who live on rice as a staple diet. Therefore, there is a need for the establishment of a validated method for the determination of fumonisins in rice at a lower level.

The difficulty in obtaining accurate analytical data on the concentrations of contaminants can be mitigated if reference materials (RMs) with known concentrations of contaminants in certain matrices of the object are available. Currently, natural or spiked RMs are available for some Fusarium toxins such as deoxynivalenol (DON) in matrices of wheat and $\operatorname{corn}^{11)}$. On the other hand, there are no RMs for Fusarium toxins in rice. Therefore, I started to prepare in-house RMs containing fumonisins in rice by following the method devised by Dr. Kenji Tanaka in the preparation of RMs containing sterigmatocystin in rice ${ }^{12)}$.

\section{Preparation of RMs containing fumonisins in rice}

Wild-type Fusarium verticillioides IFM 50193 (Research Center for Pathogenic Fungi and Microbial Toxicoses, Chiba University) or MAFF 239106 (National Institute of Agrobiological Sciences (NIAS) Genebank, Tsukuba, Japan) was used for the preparation of artificially molded Japanese rice (short-grain rice) or Thai rice (long-grain rice) contaminated with fumonisins ${ }^{13,14}$. The fungus was pre-inoculated and grown on a potato dextrose agar plate for 3 days. Thirty grams of autoclaved rice grain pre-wetted with $15 \mathrm{~mL}$ of water for three hours was served as a fumonisin producing culture medium. The fungus grown on the plate was sliced as three sections of agar and inoculated on the above medium, and kept for 2 weeks at $25{ }^{\circ} \mathrm{C}$ until they accumulate over $50 \mathrm{ppm}$ of fumonisins in the rice culture medium. The culture was ground with blank rice at the ratio of 1:10 in a ShakeMaster (Biomedical Sciences, Tokyo, Japan) equipped with a container of $400 \mathrm{~g}$ capacity to make a fine powder. This procedure was repeated to obtain 2,000 $\mathrm{g}$ of powdered rice containing three-levels of FB1 (ca. 5 ppm of FB1 in Japanese rice, ca. 0.5 ppm of FB1 in Japanese rice, and ca. 0.1 ppm of FB1 in Thai rice). Randomly picked up samples of $5.0 \mathrm{~g}$ each were extracted in a $50 \mathrm{~mL}$ tube with a screw cap using $40 \mathrm{~mL}$ methanol-water solution $(3+1, \mathrm{v} / \mathrm{v})$ by vigorous vortexing for $1 \mathrm{~min}$ using a Vortex Genie2 (Scientific Industries, NY). The supernatant was purified using a strong anion exchange (SAX) - solid phase extraction (SPE) cartridge and analyzed by HPLC-FL as described in the AOAC Official Method 995.15 ${ }^{15}$, which is a validated method for the analysis of fumonisins in corn. Homogeneity of artificially prepared fumonisin-contaminated rice powder was confirmed by the IUPAC protocol (Table 1) ${ }^{13,16)}$ or using one-way analysis of variance ${ }^{14)}$. The powder samples that passed the homogeneity test were served as in-house RMs. 
Table 1. Homogeneity data of FB1 of artificially prepared fumonisin-contaminated rice

\begin{tabular}{|c|c|c|c|c|}
\hline \multirow[b]{2}{*}{ Sample ID } & \multicolumn{2}{|c|}{ Low-level contaminated rice (ppb) } & \multicolumn{2}{|c|}{ High-level contaminated rice (ppb) } \\
\hline & Replicate 1 & Replicate 2 & Replicate 1 & Replicate 2 \\
\hline 1 & 310 & 338 & 4,210 & 4,060 \\
\hline 2 & 338 & 305 & 3,620 & 3,550 \\
\hline 3 & 295 & 370 & 4,020 & 4,250 \\
\hline 4 & 299 & 339 & 3,960 & 4,080 \\
\hline 5 & 310 & 331 & 3,700 & 4,080 \\
\hline 6 & 371 & 312 & 4,400 & 4,230 \\
\hline 7 & 328 & 331 & 3,540 & 4,300 \\
\hline$S a m^{2}$ & \multicolumn{2}{|c|}{ U } & \multicolumn{2}{|c|}{0.024266667} \\
\hline critical & \multicolumn{2}{|c|}{0.001330819} & \multicolumn{2}{|c|}{0.086372} \\
\hline$S a m^{2}<$ critical? & \multicolumn{2}{|c|}{ ACCEPT } & \multicolumn{2}{|c|}{ ACCEPT } \\
\hline
\end{tabular}

\section{Extraction, purification, and detection of fumonisins in rice}

Using in-house RMs described above, each procedure of analysis was examined. The value of FB 1 in the in-house RMs was compared for different extraction volumes and submergence periods. Generally, for the extraction of fumonisins from food samples, two-fold of solvent volume (for example, $100 \mathrm{~mL}$ of methanolwater $(3+1, \mathrm{v} / \mathrm{v})$ to $50 \mathrm{~g}$ of corn) has been used as an extraction procedure because this ratio was adopted by the AOAC Official Method $995.15^{15)}$. However, it was found that this extraction ratio was not sufficient for fumonisins in Japanese rice and five-fold of solvent volume (for example, $50 \mathrm{~mL}$ of methanol-water $(3+1, \mathrm{v} /$ v) to $10 \mathrm{~g}$ of Japanese rice) was required for sufficient extraction ${ }^{13)}$. In the Thai rice matrix, six-fold, eightfold and ten-fold solvent volumes were compared for the extraction of $10 \mathrm{~g}$ of Thai rice. It was found that significant higher value of FB1 was obtained in eight-fold solvent volume $(80 \mathrm{~mL})$ than in six-fold solvent volume $(60 \mathrm{~mL})$, while no significant difference was observed between eight-fold solvent volume $(80 \mathrm{~mL})$ and ten-fold solvent volume $(100 \mathrm{~mL})(\text { Table } 2)^{14)}$. Another extraction procedure was adopted using submergence in water prior to the addition of methanol, which was effective in the extraction of fumonisins in Japanese rice ${ }^{13)}$. This submergence procedure was originally devised by Dr. Machiko Saka, an expert in pesticide analysis, and proved to be effective with a dramatic increase in the value of FB1 in the matrix of Thai rice (Table 3$)^{14)}$.

The recoveries of FB1 and FB2 spiked in blank Thai rice are shown in Fig. 1. Spike and recovery tests were done in triplicate at a $0.2 \mathrm{ppm}$-spiked level. Considering the result in Table 2 , the eight-fold volume of

Table 2. Effect of extraction volume on the value of FB1 in in-house RM

\begin{tabular}{cc}
\hline Extraction solvent volume & FB1 concentration $(\mathrm{ppm})(\mathrm{n}=3)$ \\
\hline $60 \mathrm{ml}$ & $0.14 \pm 0.006^{\mathrm{a}}$ \\
$80 \mathrm{ml}$ & $0.18 \pm 0.006^{\mathrm{b}}$ \\
$100 \mathrm{ml}$ & $0.16 \pm 0.001^{\mathrm{ab}}$ \\
\hline
\end{tabular}

$\mathrm{a}, \mathrm{b}$ Mean values that do not share superscript letters in the corresponding columns were significantly different $(P<0.05)$. 
Table 3. Effect of submergence period on the value of FB1 in in-house RM

\begin{tabular}{cc}
\hline Submergence period & FB1 concentration $(\mathrm{ppm})(\mathrm{n}=3)$ \\
\hline $0 \mathrm{~min}$ & $0.17 \pm 0.006$ \\
$30 \mathrm{~min}$ & $0.32 \pm 0.01^{*}$ \\
\hline
\end{tabular}

*Mean values in the corresponding columns were significantly different $(P<0.05)$.

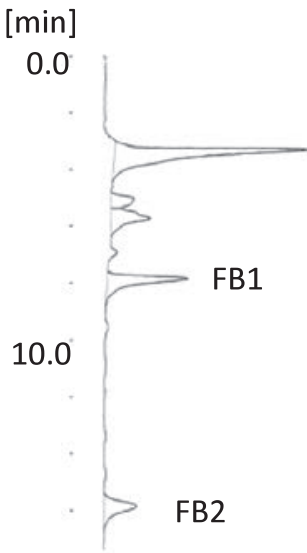

A

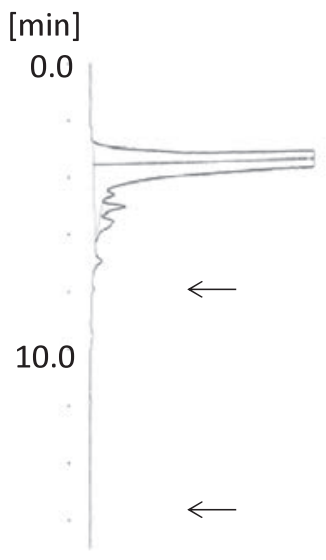

B

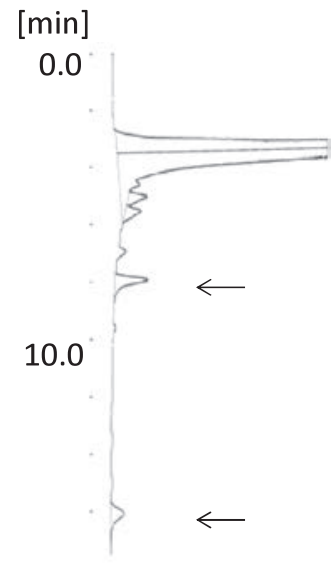

C

Figure 1. HPLC-FL chromatogram of fumonisins. A: Standard solution of FB 1 and FB 2. B: The purified extract of 0-min-submerged Thai rice (spiked FB1 and FB2 at 0.2 ppm level). C: The purified extract of 30-min-submerged Thai rice (spiked FBland FB2 at $0.2 \mathrm{ppm}$ level).

extraction solvent was adopted and the effect of submergence was evaluated. As shown, submergence was also benefitical (Figure 1C). On the other hand, without submergence, the recoveries of FB1 and FB2 were below $30 \%$ and undetectable (Figure 1B).

For the clean-up step for fumonisins, two kind of SAX-SPE cartridge, Sep-Pak Accell Plus QMA (Waters, CA) and Bond Elut SAX (Varian, CA) were used, and both were shown to work well ${ }^{13,14}$.

Detection was performed using two apparatus. The first was high-performance liquid chromatography with fluorescence detection (HPLC-FL) described in the AOAC Official Method 995.15 ${ }^{15}$. The second was liquid chromatography with tandem mass spectrometry (LC-MS/MS), which achieved a ten-times lower limit of detection (LOD) than HPLC-FL ${ }^{13)}$. HPLC-FL conditions: A conventional C18 column (L-column, CERI, Japan) and a mobile phase composed of methanol- $0.1 \mathrm{M}$ sodium phosphate monobasic $(77+23, \mathrm{v} / \mathrm{v})$ (adjusted to pH 3.3 with $o$-phosphoric acid) were used with a flow-rate of $1.0 \mathrm{~mL} / \mathrm{min}$ at $40{ }^{\circ} \mathrm{C}$. Injection was made by programming with precolumn derivatization so that $10 \mu \mathrm{L}$ of fluorescent reagent mixture $(0.3 \mathrm{M}$ $o$-phthalaldehyde in methanol- $0.1 \mathrm{M}$ disodium tetraborate - 2-mercaptoethanol $(1+5+0.05, \mathrm{v} / \mathrm{v} / \mathrm{v})$ and $10 \mu \mathrm{L}$ of sample solution was mixed followed by injection of $5 \mu \mathrm{L}$. Fluorescence was measured with excitation at $335 \mathrm{~nm}$ and emission at $440 \mathrm{~nm}$. LC-MS/MS conditions: A semi-micro C18 column (Pegasil ODS, Senshu Scientific, Japan), and gradient mobile phases composed of mobile phase A $(0.1 \% \mathrm{HCOOH}$ in $20 \mathrm{mM}$ ammonium acetate) and mobile phase $\mathrm{B}$ (acetonitrile) were used at a flow-rate of $0.3 \mathrm{~mL} / \mathrm{min}$ at $40{ }^{\circ} \mathrm{C}$. The linear gradient of A and B was performed in $25 \mathrm{~min}$ (10\% B at $0 \mathrm{~min}, 70 \% \mathrm{~B}$ at $17-19 \mathrm{~min}, 10 \% \mathrm{~B}$ at $20-25$ 
min). The injection volume was $5 \mu \mathrm{L}$ and the MS/MS monitoring ions were: $722.5 / 334.1$ (FB1), 706.4/336.1 (FB2), and 706.4/336.2 (FB3). The LOD values achieved by HPLC-FL were $50 \mathrm{ppb}$ for FB1 and $80 \mathrm{ppb}$ for FB 2 and FB 3, whereas the LOD values achieved by LC-MS/MS were 5 ppb for FB 1, FB 2 and FB 3 . In addition, LC-MS/MS does not require derivatization of extracted fumonisins ${ }^{17)}$.

Consequently, using the in-house RMs with spike and recovery tests, the following three procedures were identified as a practical analytical method for fumonisins in rice.

1) Extraction: 30 min submergence before extraction and increased solvent volume was effective. For Japanese rice, to $10 \mathrm{~g}$ of rice grain sample, first add $12.5 \mathrm{ml}$ of water, wait for $30 \mathrm{~min}$, and add $37.5 \mathrm{ml}$ of methanol to extract ${ }^{13)}$. For Thai rice, to $10 \mathrm{~g}$ of rice grain sample, first add $20 \mathrm{ml}$ of water, wait for $30 \mathrm{~min}$, and add $60 \mathrm{ml}$ of methanol to extract ${ }^{14)}$.

2) Purification: SAX-SPE cartridge is effective as described in the AOAC Official Method $995.15^{15}$.

3) Detection: HPLC-FL is useful for routine analysis, but LC-MS/MS is more suitable for detecting trace levels of contamination.

\section{Detection of trace levels of FB1 and FB2 naturally contaminating Japanese rice}

Although contamination of rice with fumonisin has been reported in the United States ${ }^{10)}$ and extensively studied in $\mathrm{EU}^{18}$, little information has come from Asia. Rice seed samples putatively infected with Fusarium in Japan were examined for the level of fumonisins contained in each subsample ${ }^{19}$ ) using the practical analytical method described above. A rice seed sample (ca. $500 \mathrm{~g}$ ) was divided into seven subsamples and ground to make aliquots of ca. $70 \mathrm{~g}$. The extract was purified and served as the sample solution for direct injection (LC-MS/MS). In this study, fumonisins in rice seeds of two subsamples were detected at levels ranging from $61 \mathrm{ppb}$ to $101 \mathrm{ppb}$ for FB1 and $11 \mathrm{ppb}$ to $27 \mathrm{ppb}$ for FB2 by LC-MS/MS (Table 4). This is the first report of naturally occurring fumonisins in Japanese rice. The level of contamination was very low, so there is a possibility that previous contamination of rice by fumonisins was overlooked by conventional extraction without submergence and by HPLC-FL detection. The amounts of fumonisins detected in this study were much lower than those generally found in corn, but monitoring of fumonisins in Japanese rice should be carried out. Further study on the retention of fumonisins during processing as well as cooking is also required to minimize exposure to fumonisins in populations who live on rice as a staple diet.

Table 4. Natural occurrence of fumonisins in Japanese rice

\begin{tabular}{|c|c|c|}
\hline \multirow[b]{2}{*}{ Sample ID } & \multicolumn{2}{|c|}{ Concentration by LC-MS/MS (ppm) } \\
\hline & FB1 & FB2 \\
\hline$\# 1$ & 0.061 & 0.011 \\
\hline$\# 2$ & $<$ LOD & $<\mathrm{LOD}$ \\
\hline$\# 3$ & $<$ LOD & $<$ LOD \\
\hline$\# 4$ & $<$ LOD & $<\mathrm{LOD}$ \\
\hline$\# 5$ & 0.101 & 0.027 \\
\hline$\# 6$ & $<$ LOD & $<\mathrm{LOD}$ \\
\hline$\# 7$ & $<$ LOD & $<\mathrm{LOD}$ \\
\hline
\end{tabular}




\section{Experimental infection of Fusarium proliferatum in rice plants}

Very recently, $F$. proliferatum strains with clear species identification were examined to determine whether $F$. proliferatum is competent to accumulate fumonisins in rice plants ${ }^{20}$. Three $F$. proliferatum strains isolated in Japan were obtained from the NIAS Genebank (Tsukuba, Japan). Isolation data on each strain (plant source, collection site, and prefecture in Japan) are as follows; MAFF 236460 (rice seed, Ishigaki, Okinawa), MAFF 236871 (rice seedling, Tsukuba, Ibaraki), and MAFF 241635 (roasted chestnut, Kobe, Hyogo). These strains were re-identified as F. proliferatum at the NIAS Genebank based on sequence analysis of the Histone $\mathrm{H} 3$ gene.

These three strains were cultured in rice seed culture and examined for the ability to produce fumonisins. As shown in Table 5 , all three strains were confirmed to be potent for producing significant amounts of fumonisins. Then, these strains were used to test for ability to infect rice plants.

Each $F$. proliferatum strain pre-cultured on SNA medium ${ }^{21)}$ at $25^{\circ} \mathrm{C}$ for $5-7$ days with near-ultraviolet light exposure (FL $20 \mathrm{~S} \cdot \mathrm{BLB}$, Toshiba, Tokyo). Conidia with mycelia were soaked with $0.01 \%$ Tween 20 solution and then filtered through a sterile gauze mesh. The conidial suspension obtained was adjusted to $10^{7}-10^{9}$ conidia/ml. A rice cultivar Kinuhikari cultivated in $18 \mathrm{~cm}$-diameter pots for $55-60$ days in a greenhouse (7-10 ears per pot, three pots for each strain) was used for infection tests. A conidial suspension of $F$. proliferatum was inoculated by spraying twice onto the ears of the rice until the ears became sufficiently wet. The first was conducted at day 7-10 after flowering, and the next was at the maturing stage. A total of $150-200 \mathrm{ml}$ of suspension was used. To promote conidial germination, the rice plants were placed at $25{ }^{\circ} \mathrm{C}$ for 2 days in a moist chamber with $100 \%$ humidity, then, transferred to a greenhouse. Successful infection and colonization were confirmed by re-isolation of inoculated fungi (Table 6). Previously, Abbas et al. pointed out the difficulty of experimental infection of $F$. proliferatum, a possible cause for Fusarium sheath rot disease found in the United States ${ }^{10)}$. The factors that elicit colonization need to be clarified in further studies.

Table 5. Production of fumonisins by $\boldsymbol{F}$. proliferatum strains cultured in rice seed culture

\begin{tabular}{lccc}
\hline \multirow{2}{*}{ Strain } & \multicolumn{2}{c}{ Concentaration in the culture $(\mathrm{ppm})(\mathrm{n}=3)$} \\
\cline { 2 - 4 } & FB1 & FB2 & FB3 \\
\hline F. proliferarum MAFF 236460 & $230+82^{\mathrm{a}}$ & $52+20^{\mathrm{b}}$ & $16+5^{\mathrm{c}}$ \\
F. proliferarum MAFF 236871 & $236+106^{\mathrm{a}}$ & $93+25^{\mathrm{b}}$ & $29+8^{\mathrm{c}}$ \\
F. proliferarum MAFF 241635 & $151+26^{\mathrm{a}}$ & $97+1^{\mathrm{b}}$ & $18+2^{\mathrm{c}}$ \\
\hline
\end{tabular}

$\mathrm{a}, \mathrm{b}, \mathrm{c}$ Mean values that do not share superscript letters in the corresponding columns were significantly different $(P<0.05)$.

Table 6. Re-isolation ratio of each strain from rice grains

\begin{tabular}{lc}
\hline \multicolumn{1}{c}{ Inoculated strain } & Re-isolation ratio \\
\hline Control (non-inoculated) & $0 \%(0 / 10)$ \\
F. proliferatum MAFF 236460 & $67 \%(4 / 6)$ \\
F. proliferatum MAFF 236871 & $100 \%(6 / 6)$ \\
F. proliferatum MAFF 241635 & $100 \%(6 / 6)$ \\
\hline
\end{tabular}


Rice grains were harvested after the rice plants wilted and served for fumonisin analysis. LC-MS/MS conditions were the same as described previously ${ }^{13)}$ with two exceptions; the mobile phase did not contain ammonium acetate and the column length was $150 \mathrm{~mm}$. The LOD values achieved by LC-MS/MS were 3 ppb for FB1 and 4 ppb for FB2 and FB3. LC-MS/MS detection demonstrated FB1 accumulation in all inoculated grains with concentrations from trace to $21 \mathrm{ppb}$ (Fig. 2).

Currently, there are no data on the survival of F. proliferatum on the sites of rice plants. Dr. Hatsuo Saitoh conducted re-isolation experiments 6 months after harvest time, and all F. proliferatum strains tested were recovered from damaged rice grains, indicating that $F$. proliferatum has strong affinity for rice grains. In addition, strong viability was confirmed in stored healthy grains (Table 7). Although the $F$. proliferatum strains tested were derived from different origins, these strains share similar features concerning survival in grains and affinity to rice plants. As Kwon et al. ${ }^{22)}$ have suggested the possibility of endophytic F. proliferatum in wheat causing seedling necrosis, further studies clarifying the affinity of $F$. proliferatum to other sites of rice plants are required. Based on the fungal recovery from inoculated rice plants, it was concluded that $F$. proliferatum was viable on brown rice for a long period, even under cold storage (Table 7 , right two columns).

At flowering, rice plants are occasionally invaded by pathogenic fungi followed by the formation of reddish sporodochia along the joint of glumes in rice ears, which is termed "Akamomi”, meaning reddish unhulled rice in Japanese. The causal fungi have been identified as F. graminearum $^{23,24)}$ and G. fujikuroi (anamorph: F. fujikuroi) ${ }^{25}$ ). Recent research has revealed that other fungi may also be responsible for “Akamomi” (Dr. Hatsuo Saitoh, unpublished). Although the infestation was not reproduced in the current study, it can be concluded that there is an evidence of fumonisin contamination in rice as a result of $F$. proliferatum infection. Surveillance for fumonisins and the adherence of $F$. proliferatum to ears of rice in the

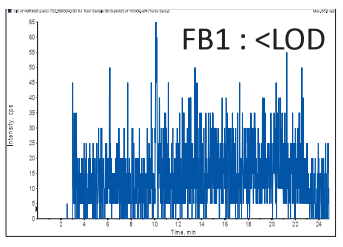

A

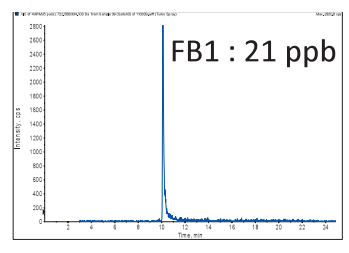

B

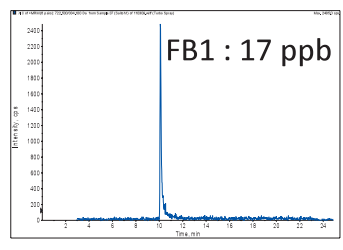

C

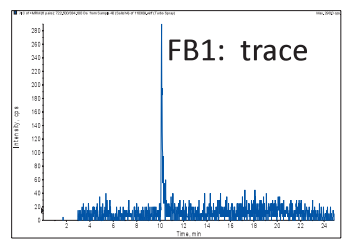

D

Figure 2. LC-MS/MS chromatogram monitored for FB1 (722.5/334.1) in extracts of rice grain of A: non-inoculated, B: inoculated with $F$. proliferatum MAFF 236460, C: inoculated with F. proliferatum MAFF 236871, and D: inoculated with $F$. proliferatum MAFF 241635.

Tabel 7. Re-isolation ratio of $F$. proliferatum strains from damaged or stored rice grains

\begin{tabular}{|c|c|c|c|c|c|c|}
\hline \multirow[t]{2}{*}{ Inoculated strain } & \multicolumn{2}{|c|}{ Grains (light damage) } & \multicolumn{2}{|c|}{ Grains (heavy damage) } & \multicolumn{2}{|c|}{ Grains (healthy) stored at $4^{\circ} \mathrm{C}$} \\
\hline & Husks & Brown rice & Husks & Brown rice & Husks & Brown rice \\
\hline Control (non-inoculated) & $0 \%(0 / 10)$ & $0 \%(0 / 10)$ & $0 \%(0 / 10)$ & $0 \%(0 / 10)$ & $0 \%(0 / 10)$ & $0 \%(0 / 10)$ \\
\hline F. proliferatum MAFF 236460 & $0 \%(0 / 10)$ & $0 \%(0 / 10)$ & $23 \%(3 / 13)$ & $15 \%(2 / 13)$ & $31 \%(4 / 13)$ & $39 \%(5 / 13)$ \\
\hline F. proliferatum MAFF 236871 & $80 \%(8 / 10)$ & $60 \%(6 / 10)$ & $77 \%(10 / 13)$ & $69 \%(9 / 13)$ & $85 \%(11 / 13)$ & $69 \%(9 / 13)$ \\
\hline F. proliferatum MAFF 241635 & $40 \%(4 / 10)$ & $40 \%(4 / 10)$ & $62 \%(8 / 13)$ & $39 \%(5 / 13)$ & $92 \%(12 / 13)$ & $100 \%(13 / 13)$ \\
\hline
\end{tabular}


paddy field will be needed to assess the natural occurrence of fumonisin contamination in Japanese rice. Furthermore, the monitoring of fumonisins in stored rice grains may also be required to prevent any undesired intake of mycotoxins.

\section{Topic II : Retention of deoxynivalenol and nivalenol during milling of wheat}

\section{Introduction}

DON is a naturally occurring mycotoxin mainly produced by Fusarium graminearum and F. culmorum, which are typical field fungi distributed widely around the world. The intake of DON by animals leads to feed refusal, reduced weight gain, and vomiting, depending on the animal species ${ }^{26,27)}$. Contamination by DON in wheat-based products is one of the most serious concerns for food and feed safety, and there exist regulatory limits for DON in many countries. In Japan, the tentative regulatory limit of DON in unprocessed wheat grain was set as $1.1 \mathrm{ppm}$ in 2001 .

Another type B trichothecene, nivalenol (NIV), is an analog of DON and often co-occurs with DON. Although similar or higher toxicity was implicated for NIV compared with DON, no regulatory limits have been placed on NIV contamination. In North America (a major area of world wheat production), NIV is not considered as important a hazard as DON, because the co-occurrence of NIV is rare. In contrast, a higher occurrence of NIV-producing Fusarium isolates was observed in Asia ${ }^{28,29)}$. Therefore, both DON and NIV are important contaminants to monitor and assess in Japan.

Because most wheat is converted to wheat flour, the milling process is a vital point to control contamination by DON and NIV. In 2008 , information about the retention of DON/NIV after harvest and during processing was very limited and focused only on $\mathrm{DON}^{30}$. Furthermore, no internationally validated simultaneous analytical methods for the quantification of DON and NIV in wheat grains and their resulting milling fractions have been described.

Therefore, test milling of artificially contaminated Japanese wheat was conducted followed by the analysis of DON/NIV during processing using an in-house validated analytical method. The distribution of DON/NIV in milling fractions of a Japanese wheat cultivar was investigated ${ }^{31}$, and also, ergosterol concentrations in the milling fractions were analyzed as an index of fungal biomass to elucidate the relationship between DON/NIV accumulation and fungal invasion into the grain ${ }^{32)}$.

\section{Wheat samples and flour preparation}

Wheat grains of a Japanese wheat cultivar, Chikugoizumi (a cultivar for noodle making), was provided. They were planted, artificially inoculated with $F$. graminearum and harvested in an experimental field in Kumamoto, Kyushu, Japan in 2008. A mixture of two F. graminearum strains was used for the inoculation: MAFF 101551 (a DON-producing strain) and MAFF 240548 (a NIV-producing strain) (NIAS Genebank, Tsukuba, Japan). Preparation of macroconidial suspension stock and spray inoculation of the suspension onto wheat plants were performed as described previously ${ }^{33)}$. The grains after harvest were combined, cleaned, and stored at $4{ }^{\circ} \mathrm{C}$ until milling.

Cleaned grains ( 3 to $5 \mathrm{~kg}$ ) were tempered to $14.5 \%$ to $16.0 \%$ water content depending on the cultivar 
for one night before milling. Milling was performed using a laboratory mill (model MLU-202, Bühler AG, Uzwil, Switzerland) equipped with three break rolls and three middling (reduction) rolls to produce eight fractions: 1B, 2B, 3B, 1M, 2M, 3M, bran, and shorts (Fig. 3). Milling conditions such as the spacing of the rolls and milling speed were based on the manufacturer's instructions. Each fraction was collected separately and mixed to homogeneity. Patent flour (aimed for human consumption) was made by mixing fractions 1B, $1 \mathrm{M}, 2 \mathrm{~B}$, and $2 \mathrm{M}$, and low-grade flour was made by mixing fractions $3 \mathrm{~B}$ and $3 \mathrm{M}$. Patent flour, low-grade flour, bran, and shorts were stored at $4{ }^{\circ} \mathrm{C}$ until analysis.

\section{In-house validation of DON and NIV analysis: spike and recovery tests}

The analytical method was based on a Japanese official method ${ }^{34)}$. Ten grams of the each sample (ground whole grain, milling fractions, raw noodles and boiled noodles) was weighed in a $300 \mathrm{~mL}$ Erlenmeyer flask with a ground-in stopper, and DON and NIV were extracted with $40 \mathrm{~mL}$ of acetonitrile-water $(85+15, \mathrm{v} / \mathrm{v})$. Ten milliliters of the supernatant or solution was transferred to a MF-T1500 multifunctional column (Autoprep, Showa Denko, Tokyo, Japan). The first $3 \mathrm{~mL}$ of eluate was discarded, and the following 5 $\mathrm{mL}$ was collected in a new test tube. A $2.0-\mathrm{mL}$ aliquot of the collected solution was evaporated at $40{ }^{\circ} \mathrm{C}$ under a gentle flow of nitrogen. The residue was dissolved in $0.5 \mathrm{~mL}$ of acetonitrile-methanol-water $(1+1+18, \mathrm{v} /$ $\mathrm{v} / \mathrm{v}$ ) and served as the sample solution for direct injection into the high-performance liquid chromatography with ultraviolet detection (HPLC-UV) apparatus. Analysis was done in triplicate $(n=3)$. HPLC-UV analysis was performed using a conventional C18 column and the mobile phase composed of methanol-water $(1+4$,

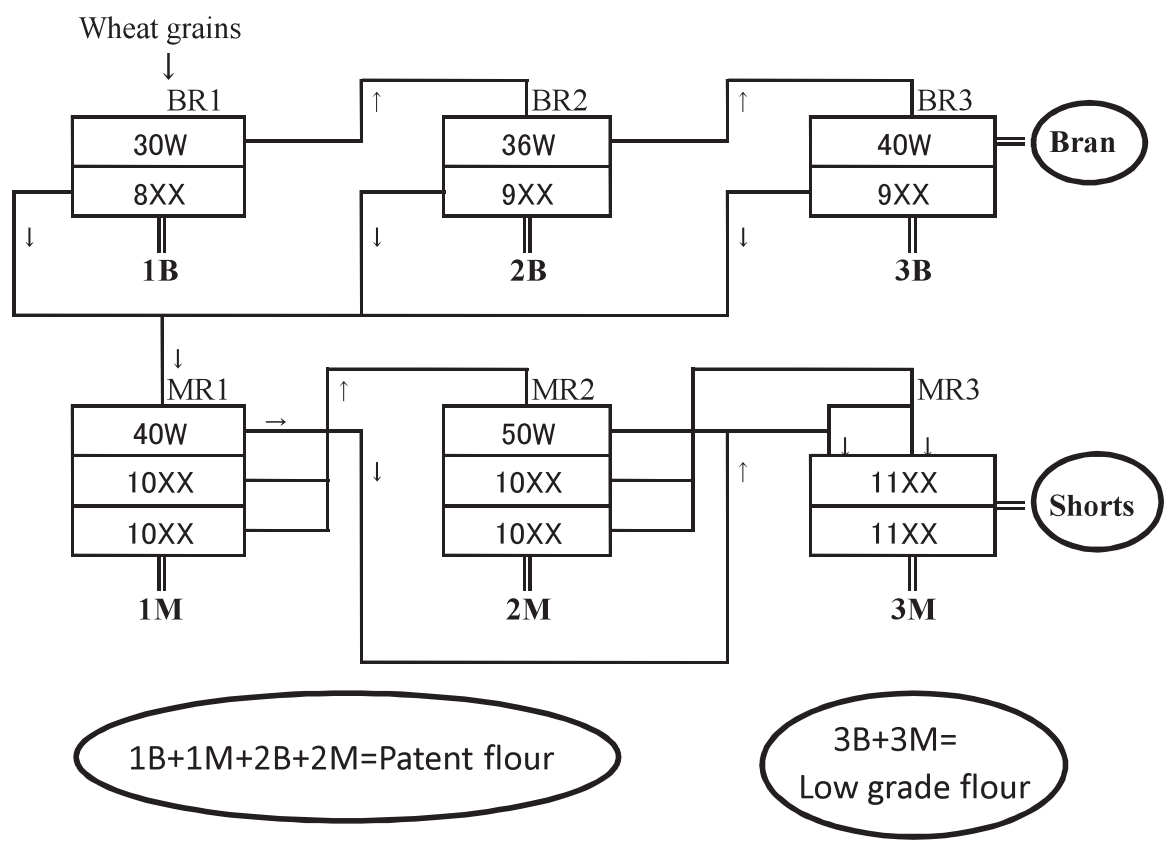

Figure 3. Milling diagram of grain from a Japanese wheat cultivar Chikugoizumi. BR: break roll, MR: middling roll, 1B, 2B, $3 \mathrm{~B}$ : break roll flour fractions, $1 \mathrm{M}, 2 \mathrm{M}, 3 \mathrm{M}$ : middling (smooth) roll flour fractions. 
Tabel 8. Recovery of toxins spiked into uncontaminated Chikugoizumi wheat

\begin{tabular}{cccc}
\hline Sample (spiked analyte) & Spiked amount (ppm) & Mean recovery (\%) & RSD \\
\hline Grain (DON/NIV) & 0.2 & $107.0 / 103.5$ & $5.9 / 4.4$ \\
& 0.5 & $96.0 / 79.5$ & $3.7 / 3.6$ \\
Patent flour (DON/NIV) & 1.0 & $100.3 / 80.7$ & $1.2 / 2.2$ \\
& 0.2 & $107.8 / 83.2$ & $4.9 / 4.3$ \\
Low-grade flour (DON/NIV) & 0.5 & $106.5 / 87.5$ & $1.7 / 4.9$ \\
& 1.0 & $106.3 / 79.9$ & $1.4 / 2.2$ \\
Bran (DON/NIV) & 0.2 & $106.0 / 98.5$ & \\
& 0.5 & $93.0 / 81.2$ & \\
Shorts (DON/NIV) & 1.0 & $95.7 / 90.1$ & \\
& 0.2 & $103.0 / 80.0$ & $1.3 / 1.3$ \\
& 0.5 & $94.1 / 73.7$ & $3.1 / 1.5$ \\
& 1.0 & $94.0 / 72.1$ & $2.5 / 1.2$ \\
\hline
\end{tabular}

$\mathrm{v} / \mathrm{v}$ ) at a flow-rate of $1.0 \mathrm{~mL} / \mathrm{min}$ at $40{ }^{\circ} \mathrm{C}$. The detector wavelength was $220 \mathrm{~nm}$.

The recovery of DON and NIV spiked into wheat grains and milled preparations is shown in Table 8 . Recovery values are shown as the average value of triplicate data with relative standard deviation (RSD) except for low-grade flour and shorts, in which there were insufficient amounts for triplicate analysis. A tentative limit for wheat grains in Japan has been set for DON at $1.1 \mathrm{ppm}$; therefore, spiking concentrations were set at $0.2,0.5$, and $1.0 \mathrm{ppm}$, which reflect one-fifth, one-half, and the same as the tentative limit, respectively. The toxin recoveries in Chikugoizumi wheat were between $72.1 \%$ and $123.0 \%$. All recovery percentages, except those for shorts spiked with $0.2 \mathrm{ppm}$ DON, were between 70 and $120 \%$, the range generally accepted as an in-house validation of an analytical method for spike and recovery tests. Although the percentage for shorts spiked at $0.2 \mathrm{ppm}$ DON was outside of the accepted range, the difference was small (123.0\%) and was not considered important for practical purposes. The RSD of DON/NIV recovery from wheat grains was low enough. For DON/NIV in patent flour and bran, the recovery values also were stable, with low RSDs. These results indicated that the simultaneous analytical method for DON and NIV in this Japanese cultivar (Chikugoizumi) is applicable for the practical analysis of wheat grain and milled preparations.

\section{Distribution of DON and NIV in the milling fractions of Japanese wheat}

Most previous data regarding DON retention during milling of North American and European wheat cultivars suggested that DON contamination was exclusively restricted to the outer layers of grain: bran and shorts ${ }^{30)}$. Little information is available on the retention of DON and NIV in Asian wheat cultivars during milling, and no internationally validated simultaneous analytical methods for quantification of DON and NIV in wheat grains and their resulting milling fractions have been described.

In this study, a laboratory mill that mimics industrial scale mills without a purifier was used, because this mill has been widely used in previous studies ${ }^{30}$. The concentration of DON/NIV in original grains and in milled preparations $(n=3)$ is shown in Tables 9 and 10 with the yield of each flour fraction. One is at a low 
level (NIV: $0.30 \mathrm{ppm}$, Table 9) and the other is at a medium level (NIV: $0.72 \mathrm{ppm}$ and DON: $0.90 \mathrm{ppm}$, Table 10) of contamination. No differences in milling properties were observed between grains with different toxin concentrations; they had very similar weight percentages for patent flour, low-grade flour, bran, and shorts. In contrast, the distribution of toxin was differed among the preparations. The concentration of NIV was high in bran or shorts from grain with a low level of contamination, with a two- and threefold higher value than in the original grain, respectively (Table 9). This result obtained from Chikugoizumi wheat with a low level of contamination was in agreement with the findings reported by Lee et al., indicating a higher percentage of NIV in the outer layers of the grain ${ }^{35}$. However, the result from Chikugoizumi with a medium level of contamination was not in agreement with previous findings; a relatively high percentage of DON/NIV was found only in patent flour, which is derived from inside (endosperm) of the grain (Table 10). In this sample, the percentage of DON and NIV was almost identical to that the weight percentage, suggesting that these two toxins accumulated in the whole grain homogeneously. Thus, the distribution of DON and NIV in milling fractions seems to be dependent on the contamination level in the original grains. More milling experiments will be useful for identifying the threshold DON/NIV concentration in the original grain at which DON/NIV accumulates in the whole grain.

Table 9. Milling properties of low-level contaminated sample and the distribution of analytes

\begin{tabular}{|c|c|c|c|c|c|c|c|c|c|c|}
\hline \multirow[b]{2}{*}{ Sample } & \multirow{2}{*}{$\begin{array}{c}\text { Weight } \\
(\%)\end{array}$} & \multicolumn{3}{|c|}{ Concentration (ppm) } & \multicolumn{3}{|c|}{ Mass balance* } & \multicolumn{3}{|c|}{ Distribution (\%) } \\
\hline & & DON & NIV & Ergosterol & DON & NIV & Ergosterol & DON & NIV & Ergosterol \\
\hline Grain & & - & $0.30 \mathrm{~b}$ & $3.46 \mathrm{c}$ & - & & & - & & \\
\hline Patent flour & 67.3 & & $0.13 \mathrm{a}$ & $0.61 \mathrm{a}$ & & 0.0875 & 0.4105 & & 33.6 & 14.3 \\
\hline Low-grade flour & 3.0 & & $0.15 \mathrm{a}$ & $1.89 \mathrm{~b}$ & & 0.0045 & 0.0567 & & 1.7 & 2.0 \\
\hline Bran & 27.0 & & $0.54 \mathrm{c}$ & $8.18 \mathrm{e}$ & & 0.1458 & 2.2086 & & 56.0 & 76.7 \\
\hline Shorts & 2.7 & & $0.84 \mathrm{~d}$ & $7.52 \mathrm{~d}$ & & 0.0227 & 0.2030 & & 8.7 & 7.1 \\
\hline Total & 100 & & & & & 0.26 & 2.88 & & 100 & 100 \\
\hline Mass balance rece & very & & & & & $\begin{array}{l}0.26 / 0.30= \\
0.87\end{array}$ & $\begin{array}{l}2.88 / 3.46= \\
0.83\end{array}$ & & & \\
\hline
\end{tabular}

$\mathrm{a}, \mathrm{b}, \mathrm{c}, \mathrm{d}$ Mean values that do not share superscript letters in the corresponding columns were significantly different $(P<0.05)$. *Calculated by [Weight (g) X Concentration (ppm)]

Table 10. Milling properties of medium-level contaminated sample and the distribution of analytes

\begin{tabular}{|c|c|c|c|c|c|c|c|c|c|c|}
\hline \multirow[b]{2}{*}{ Sample } & \multirow{2}{*}{$\begin{array}{c}\text { Weight } \\
(\%)\end{array}$} & \multicolumn{3}{|c|}{ Concentration (ppm) } & \multicolumn{3}{|c|}{ Mass balance* } & \multicolumn{3}{|c|}{ Distribution (\%) } \\
\hline & & DON & NIV & Ergosterol & DON & NIV & Ergosterol & DON & NIV & Ergosterol \\
\hline Grain & & $0.90^{\mathrm{b}}$ & $0.72^{\mathrm{b}}$ & $11.5^{\mathrm{b}}$ & & & & & & \\
\hline Patent flour & 66.9 & $0.86^{\mathrm{ab}}$ & $0.56^{\mathrm{a}}$ & $4.0^{\mathrm{a}}$ & 0.57534 & 0.37464 & 2.676 & 68.5 & 65.5 & 27.3 \\
\hline Low-grade flour & 3.5 & $0.80^{\mathrm{ab}}$ & $0.57^{\mathrm{a}}$ & $6.0^{\mathrm{a}}$ & 0.028 & 0.01995 & 0.21 & 3.3 & 3.5 & 2.1 \\
\hline Bran & 27.1 & $0.73^{\mathrm{a}}$ & $0.56^{\mathrm{a}}$ & $22.8^{\mathrm{c}}$ & 0.19783 & 0.15176 & 6.1788 & 23.5 & 26.6 & 63.0 \\
\hline Shorts & 2.5 & $1.56^{\mathrm{c}}$ & $1.01^{\mathrm{c}}$ & $29.5^{\mathrm{d}}$ & 0.039 & 0.02525 & 0.7375 & 4.6 & 4.4 & 7.5 \\
\hline Total & 100 & & & & 0.84017 & 0.5716 & 9.8 & 100 & 100 & 100 \\
\hline Mass balance rec & very & & & & $\begin{array}{l}0.84 / 0.90= \\
0.93\end{array}$ & $\begin{array}{l}0.57 / 0.72= \\
0.79\end{array}$ & $\begin{array}{l}9.8 / 11.5= \\
0.85\end{array}$ & & & \\
\hline
\end{tabular}

a, b, c, d Mean values that do not share superscript letters in the corresponding columns were significantly different $(P<0.05)$.

*Calculated by [Weight (g) X Concentration (ppm)] 


\section{Distribution of ergosterol in milling fractions}

Ríos et al. ${ }^{36)}$ recently reported the presence of DON-producing fungi in all the milling fractions examined, including the inner part of the grain, using DNA primers specific for a DON biosynthesis gene in a European durum wheat cultivar with a high level $(4.2 \mathrm{ppm})$ of contamination. This observation was consistent with a report based on microscopy ${ }^{37}$. After it was found that DON/NIV was readily distributed among flours for human consumption, the content of ergosterol was analyzed as an index of fungal biomass to elucidate the correlation between DON/NIV accumulation and fungal invasion in Chikugoizumi wheat.

In-house validation of the analytical method for ergosterol in wheat grains and milled preparations was conducted as for that of DON and NIV analysis described above, except for that the spiking level was 5.0, 10.0 and $15.0 \mathrm{ppm}$. An analytical method using HPLC-UV was based on the method developed by Miyagawa et al. ${ }^{38)}$. HPLC separation of ergosterol was performed using a semi-micro C 18 column (CAPCELL PAK UG120, Shiseido, Tokyo, Japan) with a mobile phase of methanol-acetonitrile-water $(49+49+2, \mathrm{v} / \mathrm{v} / \mathrm{v})$. The column heater was set at $30^{\circ} \mathrm{C}$, and the injection volume was $10 \mathrm{~mL}$. Ergosterol was detected at a wavelength of $282 \mathrm{~nm}$. Sufficient recovery values and RSDs were found in grain and all milling fractions. Therefore, the analytical method for ergosterol in this cultivar was demonstrated to be applicable for the practical analysis of wheat grain and milled preparations.

The results of ergosterol analysis were included in the right columns of analytes in Tables 9 and 10. The concentration of ergosterol was lowest in patent flour and highest in bran or shorts, indicating that most of the fungi are retained in the outer layers of grain (bran and shorts) even in highly contaminated grain. The percentage distribution of ergosterol was higher in bran than in other fractions in two cases, showing $76.7 \%$ and $63.2 \%$. On the other hand, the concentrations of ergosterol in patent flour were significantly lower than those in original grain in all cases examined, which implies the effective elimination of fungi by milling.

These results indicated that he DON/NIV contents in milled preparations did not depend on the fungal biomass. This study, for the first time, indicated that the DON/NIV concentrations did not depend on the fungal biomass present therein. Therefore, there is a possibility that even when most fungi remain in the outer layers of grain, toxins might diffuse into the endosperm. Consequently, milling is not always able to effectively remove these toxins from wheat grains.

Generally, it is said that mycotoxin removal during cooking is difficult. However, in the case of DON and NIV, the noodle cooking process (with a large amount of boiling water) seems to be an effective process because they are highly hydrophilic. The fate of DON/NIV during cooking should be clarified in further studies to evaluate the potential risk of mycotoxins in final food products.

\section{Acknowledgements}

The studies from 2001 to 2011 were supported by many researchers mainly in National Food Research Institute, Tsukuba, Japan. I am deeply grateful to my former supervisors, Dr. Tadahiro Nagata and Dr. Kenji Tanaka, for their direction and support. I am also grateful to Dr. Hatsuo Saitoh and Dr. Yoshitsugu Sugiura for their direction and collaboration. I express my sincere thanks to Dr. Takashi Nakajima, Dr. Hiroyuki Nakagawa, Dr. Michihiko Saito, Dr. Kimiko Yabe, Dr. Hiroshi Okadome, Dr. Hitoshi Nagashima and Dr. 
Ushio Matsukura for helpful suggestions. Special thanks to my family, parents-in-law and my neighbors.

\section{References}

1 ) FAO Newsroom. Rice is life. Increased, sustainable rice production key to global food security. Available online: http://www.fao.org/newsroom/en/focus/2004/36887/index.html.

2 ) Tanaka, K., Sago, Y., Zheng, Y., Nakagawa, H., Kushiro, M.: Mycotoxins in rice. Int J Food Microbiol, 119, 59-66 (2007)

3 ) Kuhlman, E.G.: Varieties of Gibberella fujikuroi with anamorphs in Fusarium section Liseola. Mycologia, 74, 759-768 (1982)

4 ) Marasas, W.F.: Discovery and occurrence of the fumonisins: a historical perspective. Environ Health Perspect, 109 (Suppl 2), 239-243 (2001)

5 ) World Health Organization. Joint FAO/WHO Expert Committee on Food Additives. Safety evaluation of certain food additives and contaminants in food: Fumonisins. In Proceedings of the 56th Meeting of the Joint FAO/WHO Expert Committee on Food Additives, World Health Organization: Geneva, Switzerland, 2001; pp. 103-279.

6 ) Rheeder, J.P., Marasas, W.F., Vismer, H.F.: Production of fumonisin analogs by Fusarium species. Appl Environ Microbiol, 68, 2101-2105 (2002)

7 ) Marasas, W.F.O.: Fumonisins: Their implications for human and animal health. Nat Toxins, 3, 193-198 (1995)

8 ) World Health Organization, International Agency for Research on Cancer. Fumonisin B 1 . In IARC Monographs on the evaluation of carcinogenic risks to humans; IARC press: Lyon, France, 2002; Vol. 82, pp. 301-366.

9 ) Hendrick, K.: Fumonisins and neural tube defects in South Texas. Epidemiology, 10, 198-200 (1999)

10) Abbas, H.K., Cartwright, R.D., Shier, W.T., Abouzied, M.M., Bird, C.B., Tice, L.G., Ross, P.F., Sciumbato, G.L., Meredith, F.I.: Natural occurrence of fumonisins in rice with sheath rot disease. Plant Dis, 82, 22-25 (1998)

11) Josephs, R.D., Derbyshire, M., Stroka, J., Emons, H., Anklam, E.: Trichothecenes: Reference materials and method validation. Toxicol Lett, 153, 123-132 (2004)

12) Tanaka, K., Sagou, Y., Nakagawa, H., Naito, S., Kushiro, M.: Preparation of a reference material containing sterigmatocystin. Food Addit Contam, 25, 1141-1146 (2008)

13) Kushiro, M., Zheng, Y., Sagou, Y., Tanaka, K., Nagata, T.: Liquid chromatographic determination of fumonisins B1, B2, and B3 in rice. Mycotoxins, 57, 95-104 (2007)

14) Awaludin, N., Nagata, R., Kawasaki, T., Kushiro, M.: Preparation of an in-house reference material containing fumonisins in Thai rice and matrix extension of the analytical method for Japanese rice. Toxins, 1, 188-195 (2009)

15) AOAC 49.5.01, AOAC Official Method 995.15. Fumonisins B1, B2, and B3 in Corn. Liquid Chromatographic Method. In Official Methods of Analysis of AOAC INTERNATIONAL, 18 th Edition; AOAC International: Maryland, MD, USA, 2008.

16) Thompson, M., Ellison, S.L.R., Wood, R.: The international harmonized protocol for the profi ciency testing of analytical chemistry laboratories. Pure Appl Chem, 78, 145-196 (2006) 
17) Kushiro, M., Tanaka, K., Miyazaki, S., Nagata, T.: Advances of liquid chromatographic determination of fumonisins; Potential mycotoxins for humans. Curr Pharm Anal, 2, 289-297 (2006)

18) Directorate-General Health and Consumer Protection: Final Report SCOOP Task 3.2.10. 485-578 (2003)

19) Kushiro, M., Nagata, R., Nakagawa, H., Nagashima, H.: Liquid chromatographic detection of fumonisins in rice seed. Rep Nat'l Food Res Inst, 72, 37-44 (2008)

20) Kushiro, M., Saitoh, H., Sugiura, Y., Aoki, T., Kawamoto, S., Sato, T.: Experimental infection of Fusarium proliferatum in Oryza sativa plants; fumonisin $\mathrm{B}(1)$ production and survival rate in grains. Int J Food Microbiol, 156, 204-208 (2012)

21) Nirenberg, H.I.: Recent advances in the taxonomy of Fusarium. Studies in Mycology, 32, 91-101 (1990)

22) Kwon, S.I., von Dohlen, C.D., Anderson, A.J.: Gene sequence analysis of an opportunistic wheat pathogen, an isolate of Fusarium proliferatum. Can J Bot, 79, 1115-1121 (2001)

23) Cattaneo, A.: Contributo allo studio dei miceti che nascono sulle pianticelle di riso. Archivio del Laboratorio di Botanica Critrogamica, Università di Pavia, 2, 117-128 (1879)

24) Miyake, I.: Studien über die Pilze der Reispflanze in Japan. Journal of the College of Agriculture, Imperial University of Tokyo, 2, 237-276 (1910)

25) Sasaki, T.: Infection of Fusarium fujikuroi toward rice ear (Japanese). Shokubutu Boueki, 29, 278-282 (1975)

26) European Commission. 2002 Opinion of the Scientific Committee on Food on Fusarium toxins. Part 6. Group evaluation of T-2 toxin, HT-2 toxin, nivalenol and deoxynivalenol. Available at: http://ec.europa. eu/food/fs/sc/scf/out123_en.pdf.

27) Rotter, B. A., Prelusky, D. B., Pestka, J. J.: Toxicology of deoxynivalenol (vomitoxin). J Toxicol Environ Health, 48, 1-34 (1996)

28) Lee, U.-S., Jang, H.-S., Tanaka, T., Toyasaki, N., Sugiura, Y., Oh, Y.-J., Cho, C.-M., Ueno, Y.: Mycological survey of Korean cereals and production of mycotoxins by Fusarium isolates. Appl Environ Microbiol, 52, 1258-1260 (1986)

29) Nakajima, T., Yoshida, M.: Mycotoxin productivity and virulence of Fusarium graminearum species complex causing Fusarium head blight on wheat and barley in the western part of Japan. Jpn J Phytopathol, 73, 103-111 (2007)

30) Kushiro, M.: Effects of milling and cooking processes on the deoxynivalenol content in wheat. Int J Mol Sci, 9, 2127-2145 (2008)

31) Thammawong, M., Okabe, M., Kawasaki, T., Nakagawa, H., Nagashima, H., Okadome, H., Nakajima, T., Kushiro, M.: Distribution of deoxynivalenol and nivalenol in milling fractions from Fusarium-infected Japanese wheat. J Food Prot, 73, 1817-1823 (2010)

32) Thammawong, M., Okadome, H., Shiina, T., Nakagawa, H. Nagashima, H., Nakajima, T., Kushiro, M.: Distinct distribution of deoxynivalenol, nivalenol, and ergosterol in Fusarium-infected Japanese soft red winter wheat milling fractions. Mycopathologia, 172, 323-330 (2011)

33) Yoshida, M., Nakajima, T., Tomooka. T.: Effect of nitrogen application at anthesis on Fusarium head blight and mycotoxin accumulation in breadmaking wheat in the western part of Japan. J Gen Plant Pathol, 74, 355-363 (2008)

34) Sugita-Konishi, Y., Tanaka, T., Tabata, S., Nakajima, M., Nouno, M., Nakaie, Y., Chonan, T., Aoyagi, M., Kibune, N., Mizuno, K., Ishikuro, E., Kanamaru, N., Minamisawa, M., Aita, N., Kushiro, M., Tanaka, K., 
Takatori, K.: Validation of an HPLC analytical method coupled to a multifunctional clean-up column for the determination of deoxynivalenol. Mycopathologia, 161, 239-43 (2006)

35) Lee, U.-S., Jang, H.-S., Tanaka, T., Oh, Y.-J., Cho, C.-M., Ueno, Y.: Effect of milling on decontamination of Fusarium mycotoxins. nivalenol, deoxynivalenol, and zearalenone in Korean wheat. J Agric Food Chem, 35, 126-129 (1987)

36) Ríos, G., Zakhia-Rozis, N., Chaurand, M., Richard-Forget, F., Samson, M. F., Abecassis, J., V. LullienPellerin, V.: Impact of durum wheat milling on deoxynivalenol distribution in the outcoming fractions. Food Addit Contam, 26, 487-495 (2009)

37) Jackowiak, H., Packa, D., Wiwart, M., Perkowski, J.: Scanning electron microscopy of Fusarium damaged kernels of spring wheat. Int J Food Microbiol, 98, 113-123 (2005)

38) Miyagawa, H., Umeda, M., Sato, T., Bandoh, S., Nakamura, S., Goto, T.: Single-laboratory validation of a method for ergosterol determination in cereals. Food Addit Contam Part A, 26, 201-206 (2009) 